\title{
Review of: "Improving usability benchmarking for the eHealth domain: The development of the eHealth UsaBility Benchmarking instrument (HUBBI)"
}

\author{
Bruno José Nievas Soriano ${ }^{1}$
}

1 Universidad de Almería

Potential competing interests: The author(s) declared that no potential competing interests exist.

Dear editors,

Thank you for the opportunity to review this article. I found this article very interesting, as it describes a bold research that I enjoyed reading, as I think that it can be useful for other researchers. Nevertheless, there are some minor aspects that I think that perhaps could be revised to better understand the external validity of the results and the conclusions obtained.

These are the following:

In Benchmark development, the authors write that «We verified the items with an independent researcher and made adjustments if necessary». It would be interesting if they offered more details about the verification, what adjustments were performed, and how they defined that it was necessary.

The authors describe that «People of 18 years or older, fluent in Dutch, were recruited to participate in this study. We recruited healthy participants via convenience sampling and a commercial panel agency situated in the Netherlands». They indeed describe that this is a convenience sample and therefore there is a considerable risk of potential selection bias. Thus, I would invite the authors to better describe the main features of the eligible population and, if possible, to assess the potential selection bias.

In Methods, the authors also describe that «One would expect that items BSP1-BSP3 have a stronger correlation with the construct 'Technical performance' than with the construct 'General system interaction' and vice versa for items BSP4 and BSP6». I think that this sentence would fit better in the Discussion, as it is not descriptive of the methods.

The authors explain that «A total of 148 people participated in this study». Although it seems an adequate number of participants, it may be low if we consider the classic rule of Kline to use 5-20 users per item. Thus, I would kindly invite the authors to describe if they performed an estimation of the required sample size. I would invite them to also assess the importance of the number of participants, regarding the results 
and the external validity of their conclusions.

They also write that «109 participants were recruited via the commercial panel agency and 37 participants via convenience sampling». Again, it is important to assess the potential selection bias, which also can affect the external validity of the conclusions.

In Limitations, I would invite the authors to assess the potential selection bias and the number of participants. 